\title{
Theoretical analysis of the focusing of acoustic waves by two-dimensional sonic crystals
}

\author{
Bikash C. Gupta and Zhen Ye \\ Wave Phenomena Laboratory, Department of Physics, \\ National Central University, Chungli, Taiwan 32054, R. O. C.
}

(Dated: October 29, 2018)

\begin{abstract}
Motivated by a recent experiment on acoustic lenses, we perform numerical calculations based on a multiple scattering technique to investigate the focusing of acoustic waves with sonic crystals formed by rigid cylinders in air. The focusing effects for crystals of various shapes are examined. The dependance of the focusing length on the filling factor is also studied. It is observed that both the shape and filling factor play a crucial role in controlling the focusing. Furthermore, the robustness of the focusing against disorders is studied. The results show that the sensitivity of the focusing behavior depends on the strength of positional disorders. The theoretical results compare favorably with the experimental observations, reported by Cervera, et al. (Phys. Rev. Lett. 88, 023902 (2002)).

PACS numbers: $43.20 .+\mathrm{g}, 43.58+\mathrm{z}, 43.90 .+\mathrm{v}$
\end{abstract}

\section{INTRODUCTION}

Photonic crystals 11. 2. (PC) are made of periodically modulated dielectric materials, and most sonic crystals [3] (SC) are made up of materials with periodic variation of material compositions. Both the photonic and sonic crystals have been studied both intensively and extensively. It has been suggested that PCs may be useful for various applications such as antennas [- 4 , optical filters [5], lasers [6], prisms (7], high-Q resonant cavities [8], wave-guides [9], mirrors [10] and second harmonic generations [11]. And SCs could be used as sound shields and acoustic filters 12, 13, 14, 15, 16, 17, 18, 19, 20]. These applications mostly rely on the existence of photonic and sonic bandgaps, and a majority of them is not concerned with the linear dispersion region well below the first gap. Thus most of earlier studies were focused on the formation of bandgaps and the propagation inhibition of waves.

Recently, the interest in the low frequency region, where the dispersion relation is linear, has just started. Since the wavelength in this region is very large compared to the lattice constant, the wave sees the media as if it were homogeneous, in analogy with wave propagation in normal media. Consequently, it has been proposed 21] that the optical lenses could be developed by use of PCs. However, no physical realization has been made so far. Along the same line of the thought, it was suggested that SCs may also be used to build acoustic lenses to converge the acoustic waves. A necessary condition to be satisfied for constructing an acoustic lens is that the acoustic impedance contrast between the SC and the air should not be large; otherwise acoustic waves will be mostly reflected. Once this condition is satisfied, the converging lens can be either convex or concave depending on whether the sound speed in the SC is smaller or greater than that in the air. Recently, it has been

*Electronic address: zhen@phy.ncu.edu.tw experimentally found 22 that the sound speed in two dimensional regular arrays of rigid cylinders in air is less than that in air and the impedance contrast between the structure and air is not large. The authors presented the physical realization of two refractive devices, namely, a Febry-Perot interferometer and an acoustic convergent lens.

In this paper we carry out numerical simulation on the focusing of acoustic waves by SCs. We wish to theoretically analyze the experimental results reported in 22 . Since the multiple scattering technique has been successfully applied earlier 14 to reproduce some experimental results on acoustic propagation and scattering in SCs, we will use this technique to study in detail about the focusing effect of acoustic waves by sonic crystals. There are several parameters associated with the SCs, such as the shapes and the arrangements of the lattice, the acoustic frequencies, and the filling factors etc. We will show how the focusing is controlled by these parameters. In addition, the sensitivity of focusing effects to positional disorders will also be studied. The paper is organized as follows. The formalism is presented in Sec. II, followed by the results and discussions in Sec. III. Here we will reproduce theoretically the experimental observations in [22. A summary concludes the paper in Sec. IV.

\section{FORMALISM}

Consider $N$ straight cylinders located at $\overrightarrow{r_{i}}$ with $i=$ $1,2, \ldots N$ to form either a completely random or regular array. An acoustic line source transmitting monochromatic waves is placed at $\overrightarrow{r_{s}}$. The scattered wave from each cylinder is responsible to the total incident wave composed of the direct wave from the source and the multiply scattered waves from other cylinders. The final wave reaching a receiver located at $\overrightarrow{r_{r}}$ is the sum of the direct wave from the source and the scattered waves from all the cylinders. Such a scattering could be solved exactly, following Twersky [23]. The essential procedure is 
presented below and the detail could be found in Ref. 15 .

The scattered wave from the $j$ th cylinder can be written as

$$
p_{s}\left(\vec{r}, \overrightarrow{r_{j}}\right)=\sum_{n=-\infty}^{\infty} i \pi A_{n}^{j} H_{n}^{(1)}\left(k\left|\vec{r}-\overrightarrow{r_{j}}\right|\right) e^{i n \phi_{\vec{r}-\overrightarrow{r_{j}}}}
$$

where $k$ is the wave number of the medium, $H_{n}^{(1)}$ is the $n$ th-order Hankel function of first kind, and $\phi_{\vec{r}-\vec{r}_{j}}$ is the azimuthal angle of the vector $\vec{r}-\overrightarrow{r_{j}}$ relative to the positive $x$ axis.

The total incident wave around the $i$ th scatterer, $p_{i n}^{i}(\vec{r})$, which is a superposition of the direct incident wave from the source and the scattered waves from all other scatterers, can be expressed in terms of the Bessel function (of first kind) as

$$
p_{i n}^{i}(\vec{r})=\sum_{n=-\infty}^{\infty} B_{n}^{i} J_{n}\left(k\left|\vec{r}-\overrightarrow{r_{i}}\right|\right) e^{i n \phi_{\vec{r}-\overrightarrow{r_{i}}}}
$$

Using the addition theorem for Bessel function, the scattered waves $p_{s}\left(\vec{r}, \overrightarrow{r_{j}}\right)$ for each $j \neq i$ can be expressed in terms of the modes with respect to the $i$ th scatterer and thus one leads to

$$
B_{n}^{i}=S_{n}^{i}+\sum_{j=1, j \neq i}^{N} C_{n}^{j, i}
$$

with

$$
S_{l}^{i}=i \pi H_{-l}^{(1)}\left(k\left|-i l \phi_{\overrightarrow{r_{i}}}\right|\right)
$$

and

$$
C_{n}^{j, i}=\sum_{l=-\infty}^{\infty} i \pi A_{l}^{j} H_{l-n}^{(1)}\left(k\left|\overrightarrow{r_{i}}-\overrightarrow{r_{j}}\right|\right) \exp \left[i(l-n) \phi_{\overrightarrow{r_{i}}-\overrightarrow{r_{j}}}\right] .
$$

Matching the usual boundary conditions for each cylinders, one obtains:

$$
B_{n}^{i}=i \pi \Gamma_{n}^{i} A_{n}^{i}
$$

where $\Gamma_{n}^{i}$ are transfer matrix relating the acoustic properties of the scatterers and the media (see Ref. [15] for the expression). All coefficients $A_{n}^{i}$ may easily be obtained by solving the Eq. (3) along with Eq. (6) and hence the total wave at any desired point outside the cylinders may be obtained as

$$
p(\vec{r})=p_{0}(\vec{r})+\sum_{i=1}^{N} \sum_{n=-\infty}^{\infty} i \pi A_{n}^{i} H_{n}^{(1)}\left(k\left|\vec{r}-\overrightarrow{r_{i}}\right|\right) e^{i n \phi_{\vec{r}-\overrightarrow{r_{i}}}} .
$$

The acoustic intensity (field) is the square module of the transmitted wave where the normalized transmission is given as $T \equiv p / p_{0}$. It is worth to mention that the total wave expressed by Eq. (7) incorporates all orders of multiple scattering and the formalism is valid for both regular and random arrangements of the scatterers.

\section{RESULTS AND DISCUSSIONS}

In the following computation, we consider regular arrays of rigid cylinders in air. The system we use is from and has been detailed in 22. There are $N$ uniform rigid cylinders of radius $a$. The area occupied by the cylinders per unit area is $\beta$. Therefore, the lattice constant can be expressed as $d=\sqrt{\left(2 \pi a^{2} / \sqrt{3} \beta\right)}$ for a triangular lattice arrangement of the cylinders. For the corresponding random arrangement of the cylinders, $d$ represents the average distance between the nearest neighbor cylinders. Unless otherwise mentioned, the lengths will be scaled by $d$ and the frequency will be scaled as $k a$, to make them dimensionless. We will mainly consider the triangular lattice and use the parameters from [22] for the filling factor, and the radii of the cylinders.

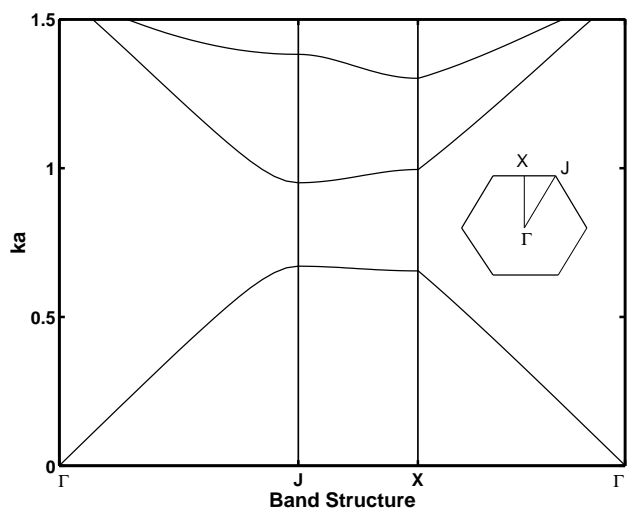

FIG. 1: Band structure for the two dimensional triangular lattice of the rigid cylinders in air.

Figure 1 reproduces the band structure for the two dimensional triangular lattice from [22. The filling fraction is 0.2024 , the radius of each cylinder is $1.5 \mathrm{~cm}$, and thus the lattice constant is $\approx 6.35 \mathrm{~cm}$. We see that the dispersion in the low frequency region is linear for both the directions. Our calculations will be confined in the linear low frequency region only.

In accordance with the experimental setup described in [22], the conceptual setup of the source and the lattice arrangement of the cylinders for the computation are shown in Fig. 2(a). The line source is denoted by S. Here the triangular arrangement in the experiment is taken, and the lenticular shape is identical to that used in the experiment 222]. The lattice constant for the lattice is $\sim 6.35 \mathrm{~cm}$, the same as considered in the experiment. The source is placed at $100 d$ away from the left side of the lattice; this distance is far enough to ensure that the incident wave on the lattice behaves as a plane wave. The wave with frequency of $k a=0.486(\sim 1700 \mathrm{~Hz})$ is incident on the triangular lattice of lenticular shape from the left, and the transmitted intensity $\left(|T|^{2}\right)$ is calculated on the right side of the lattice. The two dimensional spatial distribution of the transmitted intensity is shown in Fig. 2(b). Here the $x$ axis is a horizontal line towards 
right, and the $y$ axis is placed vertically upward. The origin is at a distance twice the radius of the cylinders to the right most cylinder, so to avoid the diverge when plotting the spatial distribution. The same arrangement of the coordinates is also used in the following figures, except for Fig. 7. We see that the focusing of the transmitted wave is evident, and is in agreement with the experimental observation [22]. Since the maximum intensity point, which we assume as the focal point, is not clear from Fig. 2(b), we have computed the variation of the field intensity along the $x$ axis with $y=0$, and also along the $y$ axis with $x=20$. The results are shown in Figs. 2(c) and 2(d). Fig. 2(c) shows that there is a peak at $x \sim 14$. The intensity variation along the $y$ axis shows a significant peak at $y=0$. Here we observe that the intensity is better confined along the $y$ axis than along the $x$ axis. The theoretical results are in a good agreement with the experimental observation.
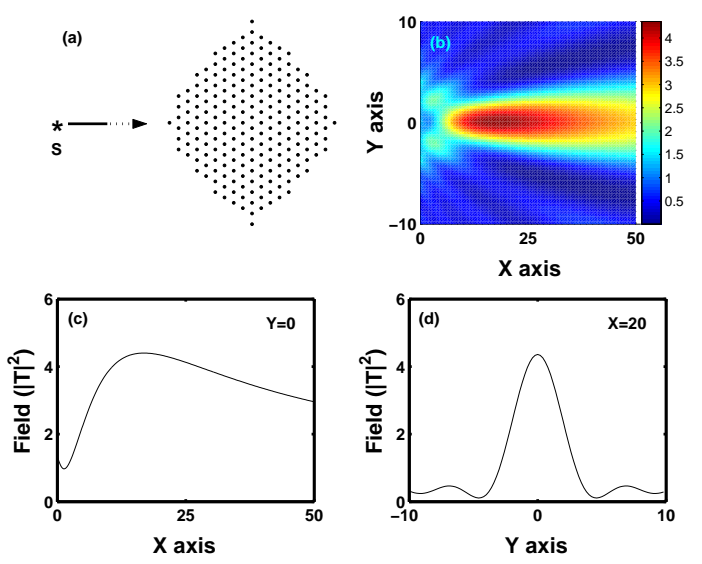

FIG. 2: (a) The line source denoted by $\mathbf{S}$ and the arrangement of cylinders; (b) two dimensional spatial distribution of the transmitted intensity $\left(|T|^{2}\right)$ on the right side of the crystal; (c) the variation of the intensity in (b) along the $x$ axis at $y=0$ and (d) the variation of the intensity in (b) along the $y$ axis at $x=20$.

In view that our numerical calculation can reproduce the experimentally observed focusing effect of sound, we are encouraged to further explore possible focusing effects with different shapes of SCs. Here we consider the lenticular shape shown in Fig. 3(a). The size of the lens along the $y$ direction is same as that for Fig. 2, whereas the size along the $x$ axis is larger. Thus, the edges of the lattice are much smoother than that in the above lens. Here the source is again kept at $100 d$ away from the left side of the lens, and other parameters are the same as used above. The transmitted intensity calculated on the right side of the lens is shown in Fig. 3(b). We see that the focusing is apparently better than that in the above $\mathrm{SC}$ of lenticular shape. We also plot the intensity variation along both the $x$ axis with $y=0$ and the $y$ axis with $x=2$. The results are shown in Figs. 3(c) and 3(d) respectively. Fig. 3(c) clearly shows that the intensity is peaked at about $x=2$ and $y=0$, then decreases more rapidly compared to that in Fig. 2(c). Fig. 3(d) also shows a narrower peak when compared with Fig. 2(d). In this case, the focal length is shorter and the intensity at the focal point is much stronger than those in the above case. Again, the intensity region is more extended along the $x$ axis compared to that along the $y$ axis.
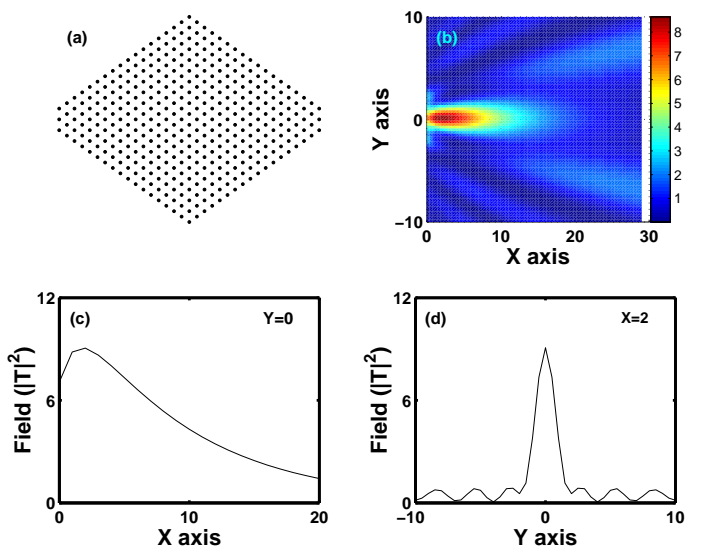

FIG. 3: (a) The arrangement of the cylinders; (b) the two dimensional spatial distribution of the transmitted intensity $\left(|T|^{2}\right)$ on the right side of the lens; (c) the variation of the intensity in (b) along the $x$ axis at $y=0$ and (d) the variation of the intensity in (b) along the $y$ axis at $x=2$.

For comparison, we have also studied the transmission through a rectangular lens shown in Fig. 目(a). The results are depicted in Fig. I(b). In this case, no unique focusing is observed. The transmitted intensity is more or less spreading all over the $x y$ plane. This can be better seen from Figs. $1(\mathrm{c})$ and $1(\mathrm{~d})$, where the intensity variations are plotted along the $x$ axis and $y$ axis respectively. These results also agree with the experimental observation [22].
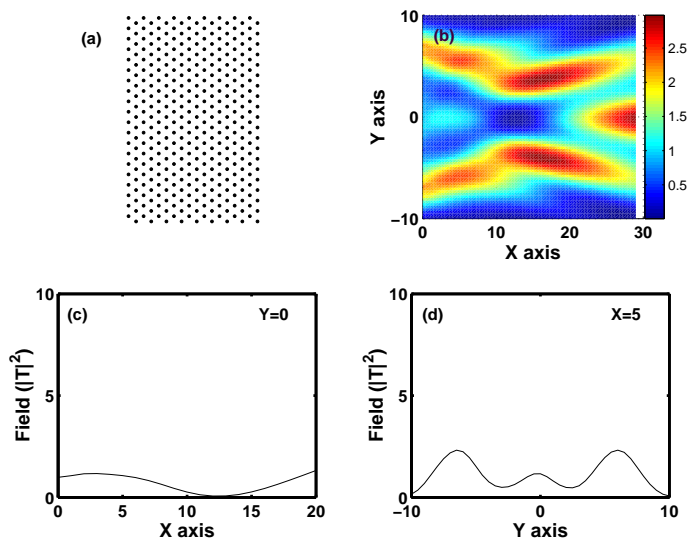

FIG. 4: (a) The arrangement of the cylinders; (b) the two dimensional spatial distribution of the transmitted intensity $\left(|T|^{2}\right)$ on the right side of the crystal; (c) the variation of the intensity in (b) along the $x$ axis at $y=0$, and (d) the variation of the intensity in (b) along the $y$ axis at $x=5$.

We also performed the similar calculations for the lat- 
tices with the regular square arrangement of the cylinders and found that the results are similar.

Figure 5(a) shows the variation of the focal length, i. e. the distance between the focal point and the center of the lattice, as a function of the filling factor. We see that the focal length $(\xi$ in $\mathrm{cm})$ decreases rapidly with the increasing filling factor $(\beta)$ up to $\beta=0.2$, then tends to saturate for higher filling factors. This decrease of the focal length with the filling factor is reasonable as the the effective sound speed in the SC decreases with increase of the filling factor, to be discussed later. The variation of the intensity at the focal point $\left(\mathrm{I}_{\max }\right)$ as a function of filling factor is also shown in Fig. 5(b). Therefore, in addition to the shapes, the filling factor is another parameter that effectively controls the focal point and focusing intensity.
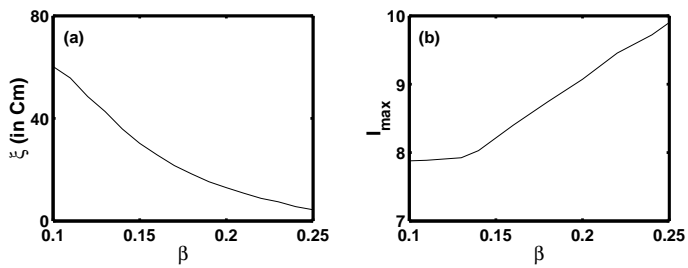

FIG. 5: (a) The variation of the focal length as a function of filling fraction $(\beta)$; and (b) the variation of Maximum intensity, $I_{\max }$ (the intensity at the focal point) as a function of the filling fraction.

From the above study on lattices of different shapes, we conclude that lattice shape is very crucial to obtain the focusing effect. The quality of focusing and the focal length could be adjusted by varying the shape of the crystal or the filling fraction.

In practice, it is useful to know the robustness of the focusing against possible disorders in the system. Here we consider the positional disorder of the cylinders. Since the wavelength is larger than the lattice constant in the present cases, one might intuitively conclude that the positional disorder has no effects. This is untrue. We have performed the calculation for three cases: the weak, moderate, and strong positional disorders. For the weak and moderate disorders, every cylinder can randomly take a position within a circle with radius of 1.4 and 15 percent of the lattice constant about its regular position, while for the strong disorder, the cylinders may take positions randomly within the boundary of the regular crystal as long as no two cylinders overlap with each other, i. e. completely random. Figs. 6(a) and (b) show the two dimensional spatial distribution of the transmitted intensities for the weak and moderate disorders. It is clear that the focusing effect is hardly affected by the presence of weak disorder. Fig. 6(c) shows the distribution of the transmitted field for the strong disorder case. Here the the intensity is highly suppressed all over the $x y$ plane and also the intensity at various places in the $x y$ plane becomes comparable to that at the maximum intensity region. Therefore, we conclude that slight or mild de- viations from perfectly regular arrangements when designing acoustic lenses will not affect the focusing drastically; therefore it will be easier to build the acoustic lens. However, the strong disorder does affect the focusing significantly. It is interesting to note that although the focusing nature is greatly reduced in the presence of strong disorders, the spatial pattern of the scattered intensity remains almost unchanged. This is because at the long wavelength region, the waves will not be able to discern the detailed structure of the crystal, and therefore the scattering pattern is mainly controlled by the shape of the crystal. The reduction in the transmitted intensity indicates that the waves are reflected backward. This is confirmed below.

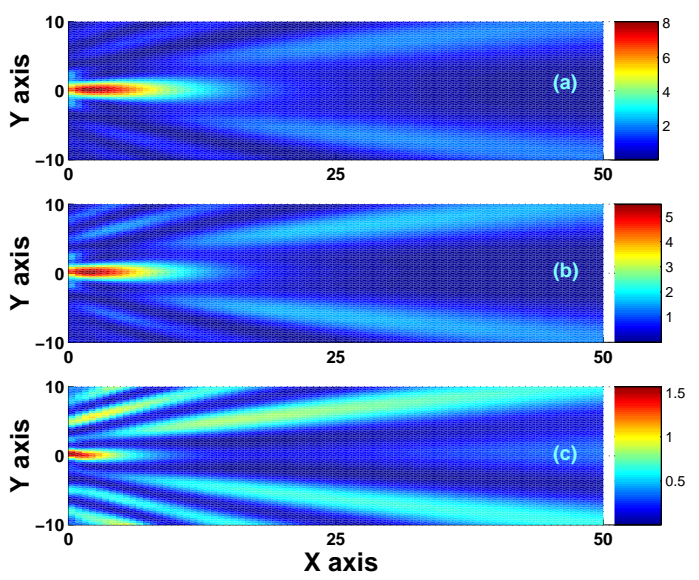

FIG. 6: Two dimensional spatial distribution of the transmitted intensity $\left(|T|^{2}\right)$ on the right side of the lattice with (a) weak, (b) moderate, and (c) strong positional disorders. The shape of the lattice and the filling factor used here are the same as that depicted for Fig. 3 .

To understand such drastic difference for the focusing between the regular lattice and the disordered lattice we plot the spatial distribution of back scattered intensities for the regular and the disordered lattices and they are shown in Fig. 7(a) and 7(b) respectively. Here the $x$ axis is a horizontal line towards right, and the $y$ axis is placed vertically upward. The origin is at a distance twice the radius of the cylinders to the most left cylinder. We observe that the distribution is symmetric for the regular case, but asymmetric for the disordered case. Compared to the forward scattering pattern for the disordered case in Fig. 6, the backscattering is more sensitive to the random configuration. This is understandable since when waves are backscattered, the travelling path will be twice that when going forward, thereby causing more interference between scattered waves. The intensity for the disordered case appears to be stronger than in the regular case. The quantitative difference is further illustrated by Figs. $7(\mathrm{c})$ and $\square(\mathrm{d})$ where the variations of back scattered intensities for both the regular and disorder cases are plotted along the $x$ axis with $y=0$ and the $y$ axis with $x=0$ respectively. While the solid curve represents 
the variation of back scattered intensities for the regular case, the dashed curve for the disordered case. Here, it is clear that the intensity strength for the disorder case is much higher. In fact, the ratio of the maximum back scattered intensity between the two cases can be as high as 15. In other words, in the disordered configuration, the transmission is highly prohibited, in agreement with the earlier observation 14. In this case, the lattice does not appear to be transparent to acoustic waves.
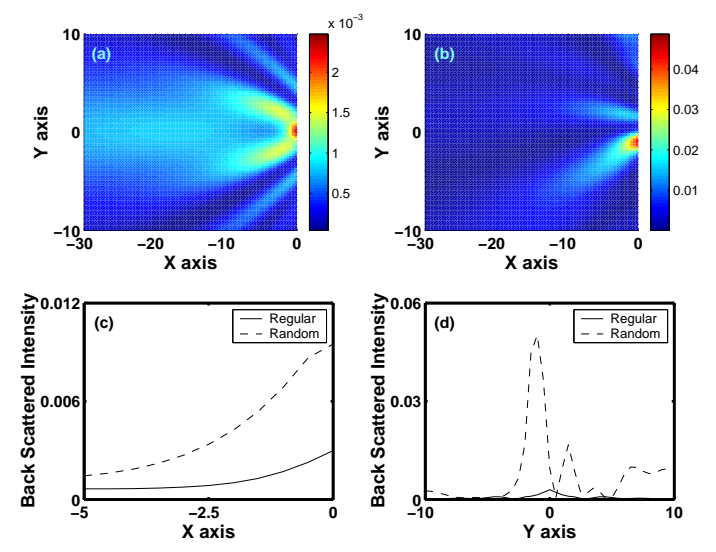

FIG. 7: Two dimensional spatial distributions of the back scattered intensities from (a) the regular lattice, and (b) one completely random configuration of the lattice; no configuration averaging is taken. The shape of the crystals for both cases is the same as that in Fig. 3 .

Finally we note on the acoustic refraction from the sonic crystal formed by the rigid cylinders in air. The focusing effect shown above is essentially, as already argued by the authors in [22], due to the reduction of the sound velocity within the SC. A simple model has been proposed by the authors to explain this reduction. Upon examination, we found that the results from the model agrees with the experimental data rather well in the low filling regime. A deviation between the theoretical and experimental data is obviously seen, referring to Fig. 2 in [22], for high filling factors. Here we would like to propose a modified model to account for the experimental data. At the frequency considered in the experiment 22] and the simulation above, the wavelength is much bigger than the lattice constant. Therefore the sonic crystal may be regarded as an effective air-filled medium, i. e. the air around each cylinder is regarded as an effective air which takes into account of the presence of other cylinders. The presence of the rigid cylinders is actually to reduce the occupation of the air per unite area without the cylinders, and is replaced by the effective air medium. This scheme may be called the self-consistent procedure. The effective mass density can be then estimated from

$$
\rho_{e}=\rho_{\text {air }}+\beta \rho_{e},
$$

where $\rho_{\text {air }}$ and $\rho_{e}$ are the densities of the air and the effective medium respectively. Thus the density of the effective medium is solved as

$$
\rho_{e}=\frac{\rho_{\text {air }}}{1-\beta} \text {. }
$$

For a given pressure, the sound speed in an air filled medium is proportional to the inverse of the square root of the density, i. e. $c \sim \sqrt{\frac{1}{\rho}}$, thus we obtain the sound speed of the sonic crystal

$$
c_{e}=c_{a i r} \sqrt{1-\beta} .
$$

Obviously, by $1-\beta \approx 1 /(1+\beta)$ for small $\beta$, the result reduces to that in 22 for small filling factors. In Fig. 8 , we show the comparison among the experimental data, and the theoretical results from the present model and that in 222]. Here we clearly see that present modified model reproduces the experimental data remarkably well, better than the original model.

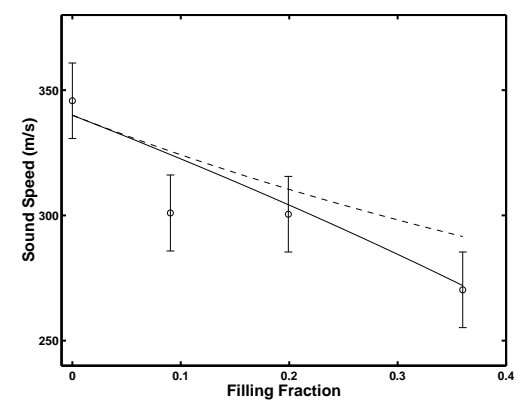

FIG. 8: The variation of sound speed in the SC with the filling fraction: The circles with error bars represent the experimental values, the dashed curve represents the theoretical estimate in [22], and the solid curve represents our estimate.

\section{SUMMARY}

Through rigorous calculations we have successfully reproduced the focusing of acoustic waves by the sonic crystal made of rigid cylinders in air, observed by the recent experiment. We have examined the focusing effect for crystals of different shapes. It has been shown that the shape of the crystal plays a crucial role on the quality of focusing. The dependance of the focal length on the filling factor is also examined. It is found that the focusing becomes brighter and the focal length decreases as the filling factor increases. The robustness of the focusing against the presence of disorders is also investigated. The results show that the robustness of the focusing behavior depends on the strength of disorders. Finally, a model describing the sound speed in the SC made from rigid cylinders arranged periodically in air as a function of filling fraction is proposed. An excellent agreement is found when the results from the model are compared with the experimental data. 


\section{Acknowledgments}

This work received support from National Science Council of Republic of China (Grant No: NSC 90-2811-
M008-004).

and R. M. de La Rue, Opt. Eng. (Bellingham) 37, 1143 (1998).

[11] J. Martorell, R. Vilaseca, and R. Corbalan, Appl. Phys. Lett. 70, 702 (1997).

[12] R. Martínez-Sala, J. Sancho, J. V. Sánchez-Pérez, J. Llinares, and F. Meseguer, Nature (London) 378, 241 (1995).

[13] J. V. Sánchez-Pérez et al., Phys. Rev. Lett. 80, 5325 (1998).

[14] Y. Y. Chen and Zhen Ye, Phys. Rev. Lett., 87, 184301 (2001).

[15] Y. Y. Chen and Zhen Ye, Phys. Rev. E, 64, 036616 (2001).

[16] C. Rubio et al., J. Lightwave Technol., 17, 2202 (1999).

[17] D. Caballero et al., Phys. Rev. E 60, R6316 (1999); Phys. Rev. B 64, 064303 (2001).

[18] W. M. Robertson and W. F. Rudy III, J. Acoust. Soc. Am., 69, 3080 (1992)

[19] L. Sanchis et al., J. Acoust. Soc. Am., 109, 2598 (2001).

[20] M. S. Kuswahs, Appl. Phys. Lett., 70, 3218 (1997).

[21] P. Halevi, A. A. Krokhin, and J. Arriaga, Phys. Rev. Lett., 82719 (1999); Appl. Phys. Lett., 75, 2725 (1999).

[22] F. Cervera et al., Phys. Rev. Lett., 88, 023902 (2002).

[23] V. Twersky, J. Acoust. Soc. Am. 24, 42 (1951).

[9] T. F. Krauss, B. Vogele, C. R. S. Stanley, and R. M. de La Rue, IEEE Photonics Technol. Lett. 9, 176 (1997).

[10] T. F. Krauss, O. Painter, A. Scherer, J. S. Roberts, 